\title{
Renal denervation in patients with resistant hypertension - single centre report
}

\author{
INGRID PRKAČIN ${ }^{1,2}$, GORDANA CAVRIĆ ${ }^{1}$,BORNA VRHOVEC ${ }^{1}$, ANA LEGOVIĆ ${ }^{1}$, PETRA \\ DRAŽIĆ ${ }^{1}$ VESNA ĐERMANOVIĆ DOBROTA ${ }^{1}$, IVA BORAS ${ }^{1}$, VINKO VIDJAK ${ }^{2,3}$ \\ ${ }^{1}$ Department of Internal Medicine, University Hospital Merkur, Zagreb, Croatia \\ ${ }^{2}$ Zagreb University School of Medicine, Zagreb, Croatia \\ ${ }^{3}$ Department of Radiology, Merkur Clinical Hospital, Zagreb, Croatia
}

Corresponding author:

Ingrid Prkacin

Clinical Hospital Merkur, I. Zajca 19, Zagreb

Phone: 0038512353-470, Fax: 0038512431-393

E-mail: ingrid.prkacin@gmail.com

\section{ABSTRACT}

Among patients with resistant hypertension, it is very important to select patients most likely to benefit from renal sympathetic denervation, because they represent a very mixed group of diagnoses.

Prior to diagnosing a patient as having resistant hypertension, it is important to document adherence and exclude whitecoat hypertension, inaccurate measurements of blood pressure and secondary causes of hypertension.

Renal sympathetic denervation has been demonstrated as an antihypertensive treatment in resistant hypertension patients with additional positive effects on glucose metabolism and insulin sensitivity in type 2 diabetes.

We report a single centre report of renal sympathetic denervation effects in a small cohort of patients with resistant hypertension and stage 2-3 chronic kidney disease. Blood pressure reduction after renal sympathetic denervation was sustained at consecutive follow-up visits one, three and six months when compared to the baseline: office systolic blood pressure was significantly lower $(144 \pm 13,140 \pm 17,141 \pm 15$ $\mathrm{mmHg}, \mathrm{p}<0.001)$.

Randomized clinical trials are required to assess the impact of the reported changes.

Key words: resistant hypertension, stepwise screening protocol, renal denervation

\section{INTRODUCTION}

Resistant hypertension is defined as blood pressure that remains above $140 / 90 \mathrm{mmHg}$ despite the continuous use of three antihypertensive agents in optimal dose, including diuretic and lifestyle changes. Resistant hypertension is associated with a hyperactive sympathetic nervous system and directly linked with a high risk of target organ damage. $(1,2)$

Increased activation of the sympathetic nervous system (SNS) is identified as an important factor in the development and progression of resistant hypertension $(\mathrm{RH})$, a condition that confers a high cardiovascular risk to the patient due to both persistent blood pressure elevation and the high prevalence of comorbidities and organ damage. $(3,4)$

Among patients with resistant hypertension, it is very important to select patients most likely benefit from renal sympathetic denervation, because patients with resistant hypertension represent a very mixed group of diagnoses. Chronic kidney disease is responsible for sympathetic nervous system hyperactivation that leads to fluid overload, aggravation of hypertension and further deterioration and loss of renal function. (3 - 5)

Despite the available pharmacological inhibition of the sympathetic nervous system, pharmacotherapy does not provide adequate effects in clinical practice. Therefore, renal sympathetic denervation (RDN), which produces multilevel inhibition of the sympathetic nervous system that triggers additional positive effects on glucose metabolism and insulin sensitivity, could be a therapeutic option for treating resistant hypertension. (5)

\section{MATERIALS AND METHODS}

The medical histories of 100 patients with resistant hypertension (from the Hospital Merkur Register) include documentation on duration, severity and progression of the hypertension; treatment adherence; response to prior medications, including adverse events; as well as current medication use, including herbal and over-the-counter medications. A biochemical evaluation of treatment-resistant hypertensive patients includes a routine metabolic profile (sodium, potassium, chloride, bicarbonate, glucose, blood urea nitrogen and creatinine) and urinalysis. The evaluation includes regular blood pressure measurements, a physical examination, and non-invasive/ invasive imaging. Before the RDN intervention antihypertensive treatment was optimized (BHS/NICE treatment algorithm), non-concordance with pharmacotherapy and secondary forms of hypertension were excluded.

Among 100 patients with resistant hypertension, we select patients (in stepwise screening protocol, multidisciplinary meeting) that were most likely to benefit from Renal sympathetic denervation (RDN). The RDN method and its risks and benefits were explained to 7 patients, and written consent was obtained. The study was approved by the local ethics committee and patients provided written informed consent to participate.

In our Centre, RDN was performed using standard radiofrequency system with ablation catheter ( $5 \mathrm{~F}$ system/6F guide catheter; SymplicityTM RDN System) inserted through the femoral artery, engaging the renal artery bilaterally.

Five to six nerve ablations of 100 second 
duration on each side were performed without any complications.

Blood pressure measurements at one, three and six month follow-up visits were compared to baseline values. We used STATISTIKA 10, 2011 software, statistically significant if $\mathrm{p}<0.001$.

\section{RESULTS}

From 100 patients referred due to resistant hypertension, more than two-thirds of the patients $(76 \%)$ did not meet the criteria of refractory resistant, or had a secondary form of hypertension (17\%).

Only seven patients with resistant hypertension (7\%) met the criteria for RDN: at baseline $2 \mathrm{M} / 5 \mathrm{~F}$, values were $62 \pm 6$ years, $184 / 106 \pm 21 / 26 \mathrm{mmHg}, 6.7 \pm 1$ for number of antihypertensive drug classes (diuretic, angiotensin-converting enzyme inhibitor or angiotensin receptor blocker, calcium channel blocker, direct vasodilator (urapidil), beta blockers, centrally acting sympatholytic (moxonidine), aldosterone antagonist (spironolactone), minoksidil), statin therapy and in $50 \%$ of patients oral hypoglicemic drugs.

At one, three and six months after the procedure, office systolic BPs were significantly lower $(144 \pm 13,140 \pm 17,141$ $\pm 15 \mathrm{mmHg}, \mathrm{p}<0.001)$. Six months after the procedure the number of antihypertensive drug classes required was $6.5 \pm 1$, with proteinuria amelioration in patients with type 2 diabetes and diabetic nephopathy (2 from 4 with DM) and stable chronic kidney disease (eGFR-MDRD: CKD stage 2 in 5F, CKD stage 3 in $2 \mathrm{M}$ ), without worsening of renal function. The office systolic blood pressure (BP) reduction after RDN was sustained at consecutive follow-up visits over 12 months when compared to the baseline.

The ABPM revealed hypertension with non-dipping $\mathrm{BP}$ pattern before $\mathrm{RDN}$ in $50 \%$ of patients. Blood pressure (BP) reduction after RDN was a 9 and $10 \%$ reduction of ABPM (sustained at follow-up visits three and six months compared to the baseline). The RDN restored the dipping BP pattern. An additional positive effect was observed on proteinuria alleviation (UAE) in two patients: after the procedure UAE was $7.9 \mathrm{mg} / \mathrm{mmol}$ in the first patient (before RDN, 8.3), and $30 \mathrm{mg} / \mathrm{mmol}$ in the second patient (before RDN, 400) with stabile values of HbA1c (6.5 and $6.9 \%$ ).

\section{DISCUSSION}

Resistant hypertension is still unrecognized as a diagnosis and insufficiently researched. $(1,2)$ Because of diagnostic procedure complexity, both doctor and patient motivation can be absent. Although the most common causes of therapeutic failure are undiscovered secondary causes of hypertension and lack of patient compliance, in about $10 \%$ of cases it can be attributed to resistant hypertension caused by a hyperactivity of the sympathetic nervous system. (3)

Numerous biological and lifestyle factors can contribute to the development of resistant hypertension: medications, volume overload, obesity, diabetes mellitus, older age, renal parenchymal and renovascular disease, primary aldosteronism, obstructive sleep apnoea, pheochormocytoma, Cushing's syndrome, thyroid diseases, and aortic coarctation. $(4,5)$

Renal physiology contributes to circadial variability of blood pressure levels. The time-related profile of blood pressure levels shows a morning increase and a deeper descent during nocturnal rest: a 10-20\% drop during the night in a healthy subject. Patients with secondary hypertension often display an abnormal circadian blood pressure profile, leading to the "non-dipper" pattern, requiring different ingestiontime dependent strategies for therapy of hypertension medications. $(6,7)$

The resistant hypertension treatment is achieved with a nonpharmacological and pharmacological approach, treating secondary hypertension causes and utilizing invasive procedures.

Initial observational studies and our results have shown that RDN is a safe and effective method of reducing office blood pressure in patients with $\mathrm{RH}$, with a positive effect on blood glucose regulation, heart and kidney function, obstructive sleep apnoea and signs of hypertensive end organ damage. $(1-3,5)$ Even though this method is promising, criticism is directed at inadequately designed and mostly observational studies without 24 hour ambulatory blood pressure measurement records, coupled with a significant variability in patient response to therapy. (8) For these reasons it is important to finally assess the effectiveness of renal denervation and the influence it might have on reducing cardiovascular morbidity and mortality.

\section{CONCLUSION}

Due to potentially beneficial reductions of multiple risk factors and safety concerns, a focused assessment of already obtained data from patients with refractory resistant hypertension after RDN intervention should be of importance.

Patients with type 2 diabetes and CKD may be the target population that would substantially benefit from RDN.

Further randomized clinical trials could be beneficial in improving both renal and cardiovascular outcomes.

A multidisciplinary meeting is necessary.

\section{REFERENCES}

1. Mahfoud F, Schlaich M, Kindermann I, et al. Effect of renal sympathetic denervation on glucose metabolism in patients with resistant hypertension: a pilot study. Circulation 2011;123:1940-6

2. Witkowski A, Prejbisz A, Florczak E et al. Effects of renal sympathetic denervation on blood pressure, sleep apnea course, and glycemic control in patients with resistant hypertension and sleep apnea. Hypertension. 2011; 58: 559-565.

3. Hering D, Mahfoud F, Walton AS, et al. Renal denervation in moderate to severe CKD. J Am Soc Nephrol 2012; 23:1250-7.

4. Prkačin I, Ožvald I, Cavrić G, Balenović D, Bulum T, Flegar-Meštrić Z. Importance of urinary NGAL, serum creatinine standardization and estimated glomerular filtration rate in resistant hypertension. Coll Antropol 2013; 37 (3): 821-825.

5. Hering D, Esler MD, Schlaich MP. Chronic kidney disease: role of sympathetic nervous system activation and potential benefits of renal denervation. EuroIntervention 2013; 9 (Suppl R): R127-35.

6. Hermida RC, Ayala DE, Portaluppi F. Circadial variation of blood presuure: the basis for the chronotherapy of hypertension. Adv 
Drug Deliv Rev 2007; 59:904-922.

7. de Jager RL, Blankestijn PJ. Pathophysiology I: the kidney and the sympathetic nervous system. Euro Intervention 2013; 9 (Suppl R): R42-7.

8. Papademetriou V, Tsioufis C, Doumas M. Renal Denervation and Symplicity HTN-3: „Dubiom Sapientiae Initium“ (Doubt is the Beginning of Wisdom). Circ Res 2014; 115 (Supl. 2): 211-214. 